\title{
A Novel Approach to Submandibular Gland Ptosis: Creation of a Platysma Muscle and Hyoid Bone Cradle
}

\author{
Robert Lukavsky ${ }^{1}$, Gary Linkov ${ }^{2}$, Christopher Fundakowski ${ }^{2,3}$ \\ ${ }^{1}$ Department of General Surgery, Temple University Hospital, Philadelphia, PA; ${ }^{2}$ Department of Otolaryngology, Temple University Hospital, \\ Philadelphia, PA; ${ }^{3}$ Fox Chase Cancer Center, Philadelphia, PA, USA
}

Submandibular gland ptosis is a common impediment to obtaining superior surgical aesthetic results in neck lift surgery. Techniques for suspending the submandibular gland have been proposed, but these procedures have the drawbacks of disturbing the floor of the mouth mucosa and periosteum. We present an approach of submandibular gland suspension for the treatment of gland ptosis by employing a platysma and hyoid bone fascia cradle. Our technique was performed on cadaveric models. The platysma muscle and hyoid bone cradle for submandibular gland ptosis was created on the left side of the neck in two cadavers. A submental incision with sharp dissection was performed to raise a supraplatysmal flap. A subplatysmal plane was developed until the submandibular gland was identified. Sutures were used to pexy the platysma to the hyoid bone periosteum and deep cervical fascia, tightening the overlying muscle and in turn elevating the submandibular gland. Submandibular gland ptosis must be corrected in order to achieve exemplary aesthetic results. Our approach of creating a cradle with the platysma and hyoid bone avoids the potential complications of previously described sling procedures, while still maintaining the integrity of the gland and surrounding tissues.

Keywords: Rhytidoplasty / Subamandibular / Cosmetics
Correspondence:

Christopher Fundakowski Department of Otolaryngology,

Temple University Hospital, 3401

North Broad Street, Philadelphia, PA

19140, USA

Tel: +1-267-746-1110

Fax: +1-215-707-1915

E-mail: Christopher.Fundakowski@

fccc.edu

Received: 12 Nov $2015 \bullet$ Revised: 31 Mar $2016 \bullet$ Accepted: 4 Apr 2016

pISSN: 2234-6163 • elSSN: 2234-6171 • http://dx.doi.org/10.5999/aps.2016.43.4.374 • Arch Plast Surg 2016;43:374-378

No potential conflict of interest relevant to this article was reported.

\section{INTRODUCTION}

Few areas of the human body symbolize youth and vigor as prominently as the neck. As we age, however, we lose the contours that help to define an aesthetically pleasing neck. Ellenbogen and Karlin [1] defined five visual criteria of the neck that are visually perceived as youthful: a distinct inferior mandibular border, a subhyoid depression, a visible thyroid cartilage bulge, a visible anterior sternocleidomastoid border, and a sternocleidomastoid-submental line angle of $90^{\circ}$. In accordance with these criteria, obtaining a distinct inferior mandibular border is crucial for ensuring the outcome of an aesthetically pleasing neck.

Submandibular gland ptosis is a common impediment to obtaining superior aesthetic results following rhytidectomy. Traditional rhytidectomy approaches, including superficial musculoaponeurotic system suspension techniques and platysmaplasty, do not address submandibular gland ptosis [2]. Several techniques of suspending the submandibular gland from the inferior border of the mandible have been described to address this dilemma $[2,3]$. However, these procedures come with the inher- 


\section{Fig. 1. Incision, platysma dissection, and submandibular gland exposure}

(A) Skin markings over the submental incision, thyroid cartilage, and cricoid cartilage. The arrow denotes the submental incision. (B) With the supraplatysmal plane elevated, the arrow demonstrates the free edge of the platysma. (C) A blunt instrument abuts the submandibular gland. Dissection was performed in a subplatysmal plane.
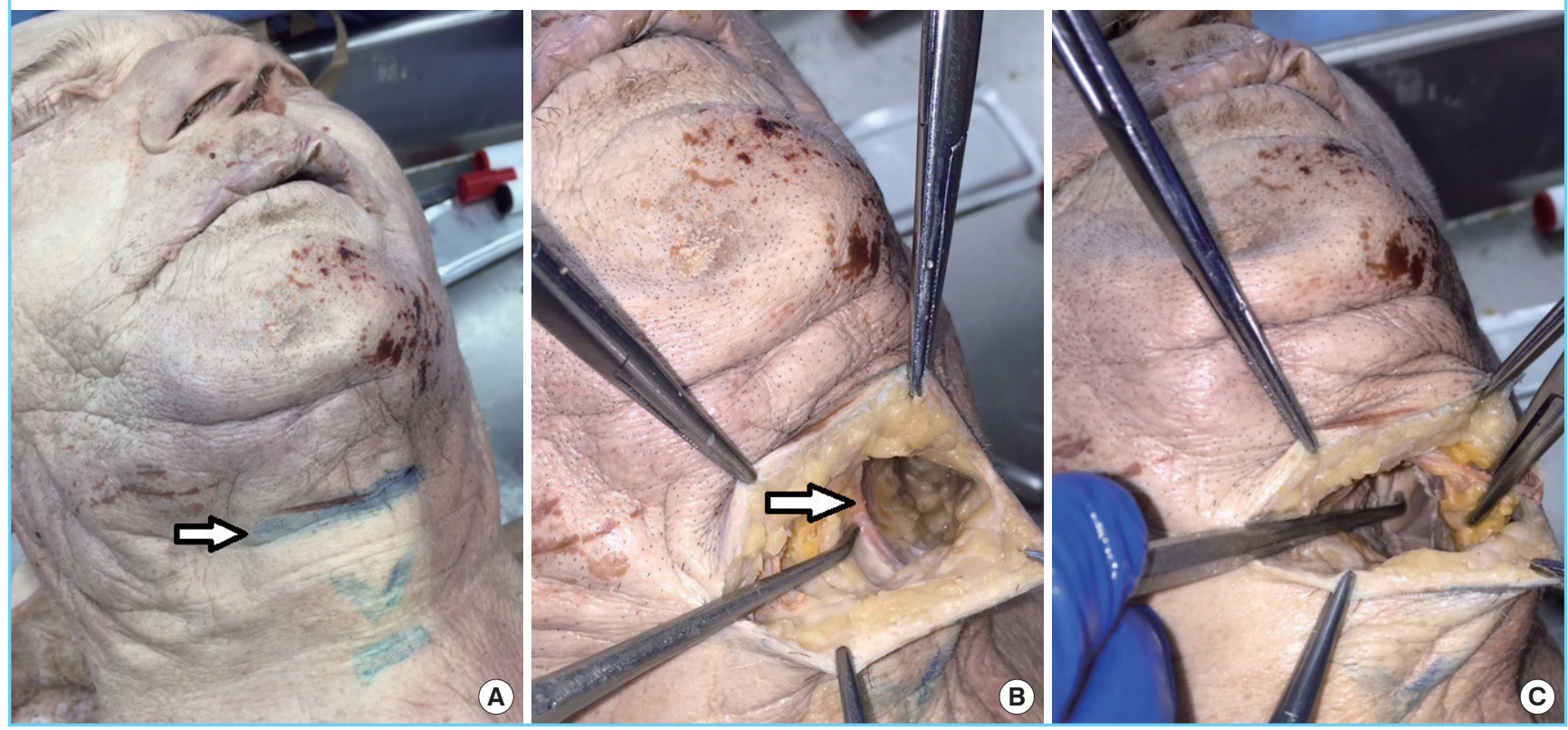

ent drawbacks of disturbing the floor of the mouth mucosa and periosteum. Additionally, gland resection is an alternative to suspending the submandibular gland, but nerve damage is a potential complication [4]. We present a novel approach of submandibular gland suspension for the treatment of gland ptosis that employs a platysma and hyoid bone cradle.

\section{IDEA}

Our proposed surgical technique is specifically directed towards correcting submandibular gland ptosis. Additional procedures would need to be used in conjunction with our approach to correct coexisting facial laxity and to achieve a more complete facial rejuvenation.

Our surgical technique was performed on two recently embalmed elderly male cadavers. A single surgeon performed the ptosis cradle procedure on the left side of the neck. Only two male cadavers were selected to be subjected to this surgical approach as a proof of concept. Future plans involve implementing our approach on patients undergoing a submentoplasty to assess the feasibility of our proposed surgical technique on submandibular gland ptosis.

Skin markings were made prior to incision over the anatomic landmarks including the hyoid bone and the thyroid and cricoid cartilages. A 3-cm submental incision was then performed at the level of the hyoid bone (Fig. 1A). While a midline scar may po- tentially become a cosmetic issue, most are hidden in natural skin creases and do not pose a problem. A supraplatysmal flap was raised sharply to the posterior aspect of the submandibular gland (Fig. 1B). The midline platysmal raphe was identified and a subplatysmal plane was developed along the inferior border of the gland overlying the lateral greater cornu of the hyoid bone (Fig. 1C). A 3-0 vicryl suture was used to pexy the platysma to the hyoid bone periosteum and overlying deep cervical fascia at the inferior border of the gland. A suture was initially passed through the fascia overlying the hyoid bone from posterior to anterior in a direction parallel to the bone (Fig. 2A). Next, the suture was passed through the platysma from deep to superficial. The suture was then advanced medially and passed back through the platysma muscle from superficial to deep and tied into place (Fig. 2B). This style of suture was placed three times, with each consecutive suture placement moving along the hyoid bone medially towards the midline (Fig. 2C, D).

\section{DISCUSSION}

Submandibular gland ptosis poses a considerable challenge in facial rejuvenation procedures. Anatomically, the submandibular gland envelops the posterior border of the mylohyoid muscle with penetration into the sublingual space [5]. Superficially, the gland is related to the facial vein and the marginal mandibular branch of the facial nerve with lateral association with the facial 

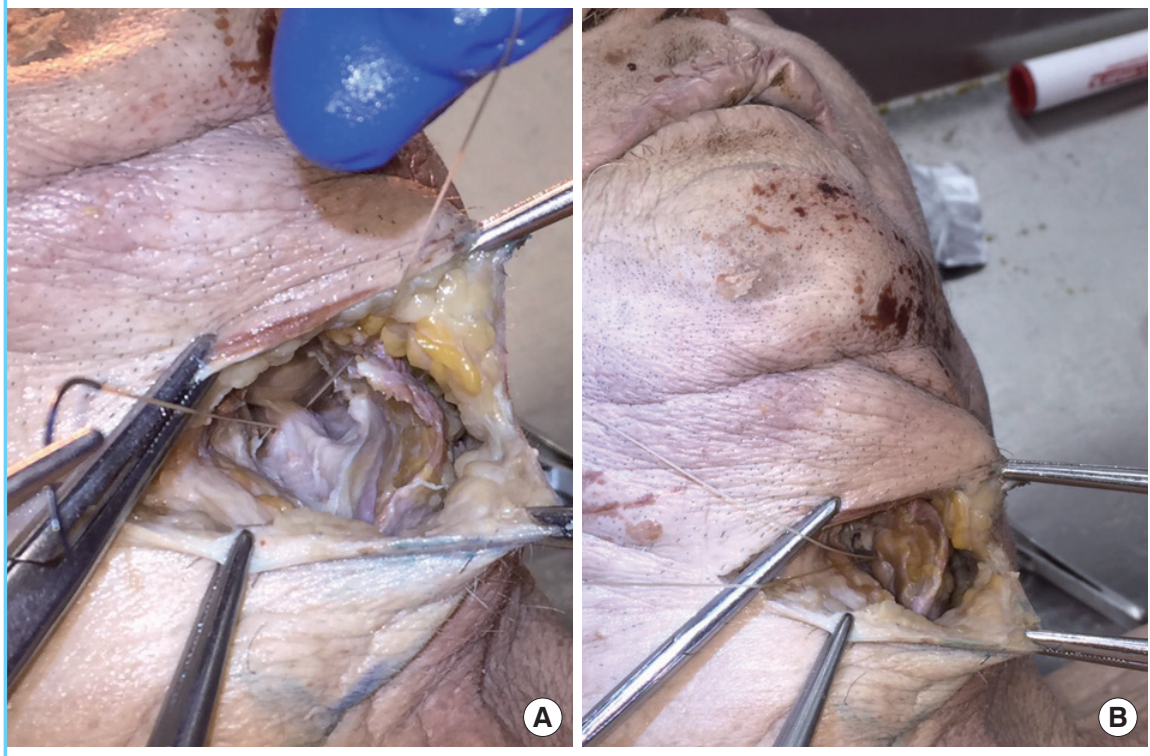

B

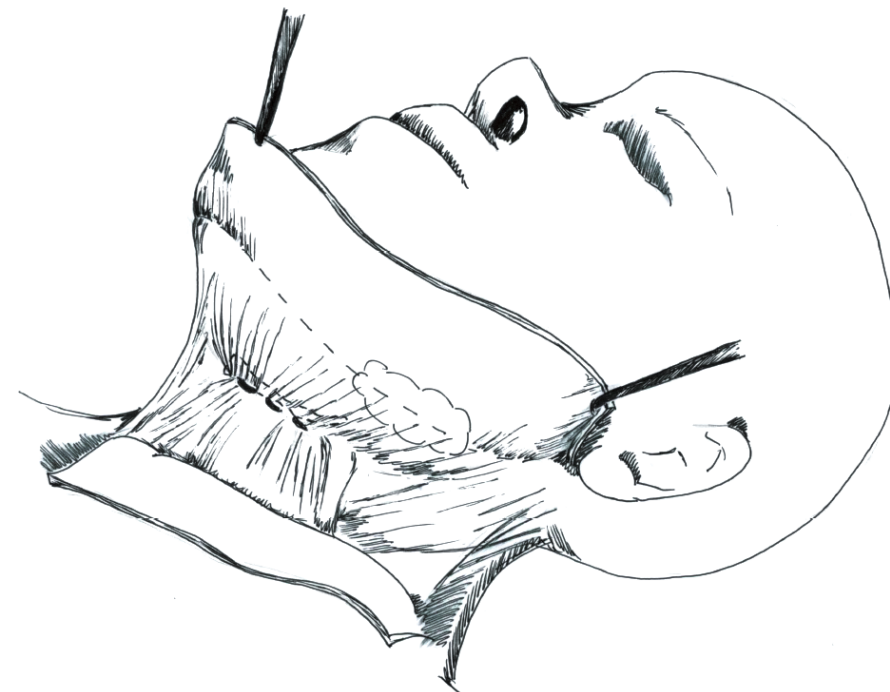

(D)
(A) A vicryl suture was used to pexy the platysma to the hyoid bone periosteum and deep cervical fascia, in essence creating a cradle for the submandibular gland. A suture was placed by first going through the deep cervical fascia and periosteum of the hyoid bone. After this, the stitch was then placed partially through the platysma muscle from deep to superficial. (B) Next, the suture was advanced medially and placed back through the platysma muscle from superficial to deep and tied into place. (C) This style of suture was placed three times with each consecutive suture placement moving more medially towards the midline. Arrows demonstrate the placement of sutures through the platysma to create the cradle with the hyoid bone in a fully dissected neck to fully illustrate the technique. (D) Image depicting the completed hyoid bone cradle as demonstrated in (C). Sutures were used to pexy the platysma to the hyoid bone periosteum and deep cervical fascia, tightening the overlying muscle and in turn elevating the submandibular gland (schematic, medical illustration performed by Catherine Kilmartin). artery [5]. The medial portion of the gland is in close contact with the hypoglossal nerve, the lingual nerve, and the submandibular ganglion of cranial nerve VII, while the deep portion of the gland is related to the lingual nerve above and the hypoglossal nerve below [5]. This extensive relationship of the gland to its surrounding neurovascular structures makes its surgical manipulation technically challenging.

In order obtain the aesthetic ideal articulated by Ellenbogen, which includes a distinct inferior mandibular border, numerous submandibular gland suspension techniques have been proposed. Sullivan et al. [2] proposed a submandibular gland suspension that relies on a suture suspension arc through the lingual mandibular periosteum with support from the capsular plication and imbrication. The benefit of this technique is that no submandibular gland tissue is excised and that only blunt dis- section is required to complete the procedure. However, an inherent limitation to this approach is violation of the floor of the mouth mucosa to complete the suture suspension, which predisposes the patient to potential infection and orocutaneous fistula formation. Sullivan et al. did not comment on the complication rate related to their technique.

Another submandibular gland suspension technique was proposed by Guyuron et al. [3] and involves drilling holes in the inferomedial border of the body of the mandible from which the suture passes in conjunction with harvested deep temporal fascia to create a basket-like structure for the gland. Similar to the Sullivan technique, Guyuron's suspension technique avoids gland resection and the complications that can arise from damage to adjacent neurovascular structures. However, unlike the Sullivan technique, no intraoral violation occurs, and the possibility for 
oral fistula is therefore limited. However, by disturbing the mandibular periosteum, Guyuron's technique theoretically predisposes the patient to possible osteomyelitis. However, the reported complications only included infected granuloma, foreign body removal due to a palpable anchor used to suspend the gland, and temporary paresthesia of the tongue [3]. Similar to Sullivan's technique, Guyuron's approach may be difficult to adopt into routine practice.

Of note, the distance of the submandibular gland from the inferior border of the mandible is variable. In a study performed by Lee et al. [6], $92.6 \%$ of patients older than 65 years of age demonstrated submandibular gland ptosis of $25 \mathrm{~mm}$ or more away from the inferior border of the mandible. In addition, numerous younger subjects in Lee's study demonstrated a distance of $20 \mathrm{~mm}$ or more between the submandibular gland and the inferior border of the mandible. By placing the submandibular gland so superiorly, Sullivan's and Guyuron's suspension techniques do not mimic the natural position of the gland and may not be feasible in routine practice.

Additionally, large hypertrophic submandibular glands can undergo resection. In a study carried out by Singer and Sullivan [4], submandibular gland resection for facial rejuvenation was proposed as a safe alternative to suspension techniques when plication alone does not provide a satisfactory outcome. Another study by de Pina and Quinta [7] further supported submandibular gland resection for patients undergoing secondary rhytidoplasty with a history of persistent gland enlargement or as a complementary procedure for patients who do not respond favorably to classic treatment with muscular plication. However, controversy exists surrounding gland resection due to the potential for nerve injury and bleeding. In the studies performed by Singer and de Pina, no reported complications or salivary fistulas were encountered $[4,7]$. Nonetheless, hesitation regarding resection of a healthy gland exists, and resection is not commonly practiced in aesthetic surgery.

Another approach to the treatment of submandibular gland ptosis involves an anterior digastric corset and lateral platysma lift. The digastric corset was conceived to address visible platysma bands noted postoperatively following platysma suspension. An additional finding was the reduction of submandibular gland ptosis due to the hammock created by the combination of the digastric corset and platysma suspension [8]. However a limitation of this approach is that the digastric corset inadvertently corrects submandibular gland ptosis with lateral traction of the gland and surrounding tissue. The submandibular gland is not secured in this technique and has the potential for recurrent ptosis in the presence of muscular laxity or redehiscence.

Our technique involves a novel approach to minimally invasive submandibular gland suspension using a hyoid bone cradle, based on familiar tissue planes and surgical exposure. By pexying the platysma to the hyoid bone, the submandibular gland is mobilized in a superior-medial position adjacent to the inferior border of the mandible. Unlike the approach proposed by Sullivan, no violation of the floor of the mouth mucosa occurs, and fistula formation is thus prevented. Additionally, the mandibular periosteum is not violated, as in Guyuron's surgical approach, so the potential for osteomyelitis is not a concern. As compared to gland resection, our approach offers the obvious benefit of maintaining a healthy submandibular gland and obviates the potential occurrence of neurovascular injury or xerostomia that may accompany resection. Finally, in comparison to Labbe's digastric corset, our approach more specifically addresses submandibular gland ptosis by placing sutures inferior to the lower margin of the ptotic gland and preventing future descent due to gravity.

Upon reflection about our technique, although a single suture could accomplish the submandibular gland repositioning, an additional suture or two may be used for reinforcement. As well, an optional final element to the procedure would include additional sutures to plicate the redundant platysma muscle, in order to avoid any cosmetically unappealing distortion in the muscle.

Despite the numerous benefits of our approach, potential drawbacks do exist. One such drawback is entrapment of the marginal mandibular nerve that runs forward below the angle of the mandible and runs beneath the platysma [9]. By pexying the platysma to the hyoid bone, the surgeon runs the potential risk of damaging the marginal mandibular nerve in the instance of a low hanging branch. A second limitation to our approach is that its efficacy may be limited for large, hypertrophic submandibular glands. In congruence with Singer and de Pina, large ptotic submandibular glands may be more amenable to resection if necessary. However, given our technique and exposure, a hypertrophic submandibular gland could theoretically undergo partial resection in addition to the creation of a platysma and hyoid bone fascia cradle to alleviate gland ptosis. Another caveat to our technique is that by securing the platysma to the hyoid to resolve gland ptosis, the traditional approach to a platysmaplasty, which involves plication of the platysmal edges medially and placing an interlocking suture from the midline to the mastoid fascia bilaterally, may no longer be feasible [10]. Another obvious limitation to our study is that it has not been performed on living tissue and the study cohort is small. Although a cadaveric model offers a host of inherent potential benefits, one of the inescapable drawbacks is rigidity of the tissues. This loss of pliability limited our ability to assess the ultimate effect of our intervention on submandibular gland ptosis, but allowed us to demonstrate that the technique is a feasible option in correcting gland 
ptosis. Current plans are underway to radiologically demonstrate the correction of ptosis in our cadaveric models and to possibly perform our technique on patients undergoing submentoplasty to assess the feasibility of our proposed surgical approach to treat submandibular gland ptosis. Although our proposed approach alleviates the aesthetically troubling effects of gland ptosis, we are not currently able to assess its effects on the patient's swallowing function. Future plans involve implementing our approach in human experiments to assess this possible effect, since swallowing is a dynamic process and cannot be evaluated in cadaveric models.

The correction of submandibular gland ptosis is an obstacle that must be overcome to achieve exemplary aesthetic results. Our approach of correcting gland ptosis by creating a cradle with the platysma and hyoid bone avoids the potential complications of previous sling procedures, while still maintaining the integrity of the gland. Future research endeavors will include performing our technique on patients undergoing submentoplasty in pursuit of a more youthful and visually appealing neck.

\section{REFERENCES}

1. Ellenbogen R, Karlin JV. Visual criteria for success in restoring the youthful neck. Plast Reconstr Surg 1980;66:826-37.

2. Sullivan PK, Freeman MB, Schmidt S. Contouring the aging neck with submandibular gland suspension. Aesthet Surg J 2006;26:465-71.

3. Guyuron B, Jackowe D, Iamphongsai S. Basket submandibular gland suspension. Plast Reconstr Surg 2008;122:93843.

4. Singer DP, Sullivan PK. Submandibular gland I: an anatomic evaluation and surgical approach to submandibular gland resection for facial rejuvenation. Plast Reconstr Surg 2003; 112:1150-4

5. Skandalakis JE, Gray SW, Rowe JS Jr. Surgical anatomy of the submandibular triangle. Am Surg 1979;45:590-6.

6. Lee MK, Sepahdari A, Cohen M. Radiologic measurement of submandibular gland ptosis. Facial Plast Surg 2013;29: 316-20.

7. de Pina DP, Quinta WC. Aesthetic resection of the submandibular salivary gland. Plast Reconstr Surg 1991;88:779-87.

8. Labbe D, Giot JP. Open neck contouring. Clin Plast Surg 2014;41:57-63.

9. Batra AP, Mahajan A, Gupta K. Marginal mandibular branch of the facial nerve: an anatomical study. Indian J Plast Surg 2010;43:60-4.

10. Giampapa V, Bitzos I, Ramirez O, et al. Suture suspension platysmaplasty for neck rejuvenation revisited: technical fine points for improving outcomes. Aesthetic Plast Surg 2005;29:341-50. 